\title{
Recombinant vaccinia viruses as vaccines
}

\author{
from F. Brown, G. C. Schild and G. L. Ada
}

The prospect of live vaccines consisting of genetically modified vaccinia virus expressing foreign genes is exciting, but important issues concerning safety and efficacy need to be resolved.

THE World Health Organization (WHO) smallpox eradication programme, involving the extensive use of vaccinia virus vaccines, resulted in the late 1970 s in the elimination of one of the world's most feared diseases. This achievement is a triumph for preventative medicine and for international collaboration in public health. The last reported case of smallpox was in 1977 . In the early 1980s, WHO recommended that the routine use of smallpox vaccine should be stopped.

Against this background, the prospect of live vaccines consisting of genetically modified vaccinia virus expressing foreign antigens arising from the work of Moss ${ }^{1}$ and $\mathrm{Paoletti}^{2}$ and their colleagues has been greeted with enthusiasm. These workers have shown that genes coding for immunogenic proteins can be inserted into vaccinia virus DNA without impairing the ability of the virus to grow in tissue culture. Moreover, experimental animals infected with vaccinia virus recombinants containing genes coding for a variety of immunizing proteins have been shown to be protected against challenge infection with the corresponding infectious agent. The studies have included recombinants which express the protective antigens of hepatitis $\mathrm{B}^{3}$, rabies ${ }^{4}$ and malaria ${ }^{5}$, agents causing diseases of major economic and social importance.

The observations so far involve only small numbers of laboratory animals; the development of vaccines to a stage where they can be evaluated in the field will inevitably take time and effort. But the potential attractions of such vaccines include not only their low cost and ease of production (once suitable recombinants are established), but ease of administration and a high degree of stability, which would put them within the reach of populations in developing countries. Even so, optimism that this approach will lead to inexpensive and effective vaccines against these and other major diseases must be tempered with the concern which stems from the known adverse reactions to vaccinia virus, themselves well documented ${ }^{6}$ during the smallpox eradication programme.

\section{Vaccinia virus}

Vaccinia virus consists of a doublestranded DNA molecule of 187 kilobases complexed with structural polypeptides and transcriptional enzymes and enclosed in a lipid envelope. Transcription and replication of the genome take place untypically in the cytoplasm of infected cells, which is attributable to the possession by the virus of enzymes necessary for nucleic acid metabolism. These include DNA and RNA polymerases, enzymes to cap, methylate and polyadenylate mRNA, and a thymidine kinase (TK); however, there are no RNA-splicing enzymes.

The virus DNA is non-infectious and so large that in vitro manipulation is difficult, for which reasons the foreign

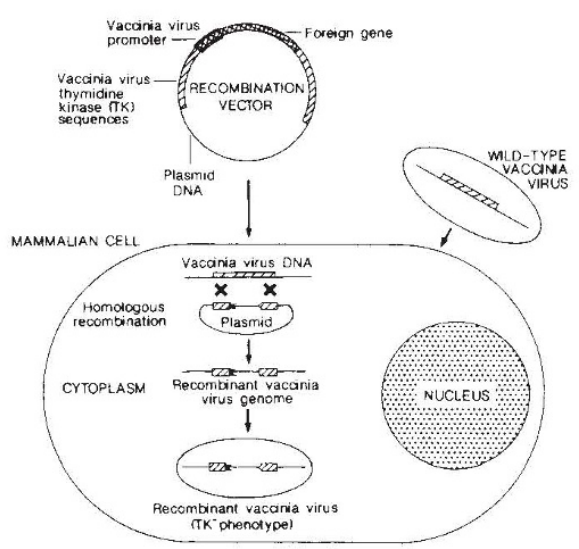

Fig. 1 Construction of vaccinia virus recombinants. The DNA sequence coding for the foreign gene is inserted into a plasmid vector downstream of a vaccinia virus promoter and flanked by vaccinia TK sequences. The resultant recombination vector is introduced into cells infected with vaccinia virus to generate a $\mathrm{TK}^{-}$ recombinant virus which expresses the foreign gene.

gene must be inserted into the virus by homologous recombination (Fig. 1). A plasmid vector in which the foreign gene is placed under the control of a vaccinia promoter and flanked by non-essential vaccinia virus DNA sequences is introduced into cells infected with vaccinia virus. Recombination between the flanking vaccinia sequences of the plasmid vector and the homologous sequences in the virus genome results in the insertion of the foreign gene into a specific site of the virus genome. Since, by arrangement, the insertion occurs at a position which is not essential for virus replication, the recombinant virus genome replicates normally and is packaged into infectious progeny virus.

Recombinant viruses generated in this way are only a small proportion of the progeny, but may be distinguished from the parent virus by selective procedures: if the foreign genes are inserted within the coding sequence of the vaccinia virus TK gene this gene is inactivated and the recombinants can be selected by plaque assay on $\mathrm{TK}^{-}$cells in the presence of 5bromodeoxyuridine. This technique has now been used to select recombinants containing genes corresponding to antigens of medical and veterinary importance, influenza ${ }^{7}$ and herpes simplex ${ }^{8}$ as well as those already listed. There is little doubt that the technique is applicable to the expression of a wide range of protein or glycoprotein antigens, while several loci other than the TK gene, also inessential for virus replication, have been identified and could be valuable in constructing new recombinant viruses.

If the vaccinia virus recombinants have the same general physicochemical properties as the parent strain, they would be expected to possess the same advantages as vaccinia virus vaccines. The parent strain has a remarkable degree of stability under field conditions, a major consideration for live vaccines intended for use in tropical countries. Moreover, the vaccine can be administered easily by the dermal route, can be produced very cheaply and provides lasting immunity after a single inoculation, which is a major consideration for vaccines intended for use in developing countries. Once a suitably engineered recombinant strain of vaccinia virus has been obtained and shown to possess suitable immunogenic properties, high technology would no longer be required in vaccine production.

But before the general use of recombinant vaccinia virus vaccines is possible, several important issues concerning the safety and efficacy of vaccines of this type, will have to be resolved.

This need has been recognized by WHO, which convened two meetings, one at the US National Institutes of Health in November 1984 and a second in Geneva in September 1985. The following important conclusions were reached.

\section{Molecular studies}

Since the efficacy of the recombinants will depend on their ability to elicit a protective immunological response, further research aimed at an increased understanding of the regulation of gene expression in vac- 
cinia virus will be required to ensure an adequate and stable level of expression of the foreign antigen. It will be necessary to define precisely the best ways of linking foreign genes to transcriptional regulatory sequences of the vaccinia virus genome and to identify the optimal sites for their insertion. The manner in which the foreign antigen is presented to the host's immune system should be investigated.

\section{Level of attenuation}

Vaccination with vaccinia virus can lead to abnormal skin eruptions, disorders affecting the central nervous system and a variety of other less severe complications. Thankfully, these severe complications are rare, so their occurrence with a genetically modified virus could be assessed only with a large-scale clinical study.

There is at present no generally accepted laboratory marker which predicts with certainty the virulence or attenuation of vaccinia virus or its recombinants for human beings. Reliable genetic markers of virulence would need to be established. Nevertheless, studies of mortality and pathogenesis in mice have been found to be of value; for example, the insertion of foreign genes in the TK gene has been found to reduce markedly the virulence of the virus ${ }^{9}$. Similar studies with other species would be valuable in telling whether this is a general phenomenon. It would be particularly useful to include primates and species such as cattle, which might be targets for immunization with recombinant vaccines. Moreover, it will be necessary, as with any attenuated virus vaccine, to study the virulence of the recombinant virus after several passages in the recipient species. Since successful immunization may require different routes of injection for different diseases, it will also be necessary to demonstrate that the recombinants are innocuous when administered by different routes.

\section{Model systems}

As there is no satisfactory laboratory animal model for assessing the virulence and efficacy of vaccinia virus, it is imperative to evaluate both in vivo and in vitro tests for their relevance in predicting the virulence for man of new recombinant strains. The results obtained should be compared with those obtained in the species for which the vaccine is intended. It will also be important to determine whether changes in host range or tissue tropism could occur as a result of the genetic modification of the virus and also by possible alterations in the viral envelope caused by insertion of a foreign gene product.

\section{Assessing priorities}

The use of vaccinia virus recombinants would be particularly advantageous for immunization against agents for which vaccines are not presently available, difficult to produce, ineffective and expensive. Moreover, the use of vaccinia virus offers the possibility of vaccination against several diseases by a single dose of a recombinant vaccine containing several gene inserts coding for different foreign antigens, but the extent to which the expression of one antigen may interfere with that of others encoded within the same genome will need to be evaluated. Studies of the immune responses of different hosts to each component antigen in multivalent recombinants will need to be carefully assessed.

In practice, it would be logical to use those vaccinia virus strains used extensively as vaccines in the WHO smallpox eradication programme and for which most information on satisfactory safety and efficacy is available. One or more wellcharacterized strains of vaccinia virus should be designated as references for use in the laboratory control and standardization of the vaccines.

\section{Vaccine production}

Vaccinia virus vaccine has been traditionally prepared in calf lymph, but this method of production is now considered by most authorities to be inappropriate. To be generally acceptable for use in man, it would be desirable to produce the vaccines in cell cultures employing the same good manufacturing practices as required for other modern live virus vaccines.

It is clear that control requirements for human vaccines using vaccinia virus as a vector for heterologous antigens would be developed progressively as more information relating to attenuation, production, safety and efficacy becomes available. Requirements applicable to live virus vaccines in general, such as the characteristics of the seed virus, use of a seed-lot system, description of the substrate and methods for production and the testing of safety, potency and stability, would be necessary. Issues peculiar to recombinant virus vaccines would thus include the description of the parent virus strain, methods for gene cloning, confirmation of the gene structure of the seed virus, the demonstration of its genetic stability and the results of clinical trials.

So that the results of research can be assessed for practical application as soon as possible, it is clear that WHO has an important role in encouraging and supporting the research work, in promoting the exchange of information and establishing technical collaboration between laboratories working on the expression of protective microbial antigens by vaccinia virus recombinants. WHO also has a role in formulating guidelines and requirements for these recombinants for the standardization, control and field use of any new vaccines developed.

While most attention has been focused on the use of vaccinia virus as the vector, similar approaches using alternative vectors, including other large DNA viruses or bacterial species, are feasible and may have advantages for some applications. For example, vectors capable of replication in the gut may be more suitable than vaccinia virus in the development of vaccines against enteric infections. Moreover, vaccinia recombinants might be used for the more efficient and safer production of antigens for the development of inactivated' vaccines, as exemplified by the work on rabies by a group at the Wistar Institute ${ }^{4}$

There is a very great potential for developing new approaches to the control of infectious disease using vaccinia virus as a vehicle, but the enthusiasm for rapid progress towards new vaccines should not be allowed to compromise the requirement for obtaining additional scientific information essential to ensure safety, efficacy and the exercise of all reasonable caution in mounting field investigations. Moreover, other approaches to vaccination are being vigorously pursued, for example controlled biosynthesis of antigens in bacterial, yeast and animal cells and site-specific mutagenesis to provide stable attenuated strains. It remains to be determined which of these approaches will provide vaccines giving optimal protection against each individual disease.

Present addresses for authors: Wellcome Biotechnology Ltd, Pirbright, Surrey GU24 ONO, UK (F.B.); National Institute for Biological Standards and Controls, Holly Hill, Hampstead, London NW3 6RB, UK (G.C.S.); John Curtin School for Medical Research, Canberra, Australia (G.L.A.).

1. Mackett, M., Smith, G. L. \& Moss, B. Proc. natn. Acad. Sci. U.S.A. 79, 7415-7419 (1982).

2. Panicali, D. \& Paoletti, E. Proc. nain. Acad. Sci. U.S.A. 79, 4927-4931 (1982)

3. Smith, G. L., Mackett, M. \& Moss, B. Nature 302, 490-495 1983

4. Kieny, M. P. et al Nature 312, 163-166 (1984).

5. Smith, G. L. et al Science 224, 397-399 (1984).

6. Fenner, F. in New Approaches to Vaccine Development (eds Bell, R. \& Torrigiani, G.) 187-196 (Schwake, Basel, 1984).

7. Panicali, D., Davis, S. W., Weinberg, R. L. \& Paoletti, E. Proc. natn. Acad. Sci. U.S.A. 80, 5364-5368 (1983).

8. Cremer, K., Mackett, M., Wohlenberg, C., Notkins, A. L. \& Moss, B. Science 228, 737-740 (1985).

9. Buller, R. M., Smith, G. L., Moss, B., Cremer, K. \& Notkins, A. L. in Vaccines 85 (eds Lerner, R. A., Chanook, R. M. \& Brown, F.) 163-167 (Cold Spring Harbor Laboratory, New York, 1985) 